\title{
Comment comprendre la mesure du spin et l'expérience EPR-B ? Une interprétation causale
}

\author{
Michel Gondran ${ }^{1}$ \\ Alexandre Gondran ${ }^{2}$ \\ (1) Académie Européenne Interdisciplinaire des Sciences, Paris, France \\ (2) École Nationale de l'Aviation Civile, Toulouse, France
}

\section{Résumé}

Le spin est un degré de liberté interne à toute particule qui n'a pas d'équivalent en mécanique classique. Pour comprendre sa réalité, nous revenons, dans cet article, sur l'expérience de Stern et Gerlach qui a mis en évidence en 1922 l'existence du spin 1/2 de l'électron. Cette expérience est l'archétype de la mesure en mécanique quantique. Elle synthétise le problème dit de la mesure (chat de Schrödinger). La résolution spatiale et temporelle de l'équation de Pauli est nécessaire pour saisir les nombreuses subtilités de cette expérience. Notamment cela contraint à considérer l'extension spatiale de la fonction d'onde. On montre que la théorie de de Broglie-Bohm (dBB) qui définit une réalité sous-jacente à la mécanique quantique habituelle en ajoutant au formalisme des variables cachées (positions des particules) donne une explication causale et réaliste de cette expérience. La version de David Bohm de l'expérience d'Einstein-Podolsky-Rosen (EPR-B) qui met en évidence la non-localité sur une paire de particules intriquées par leur spin utilise des appareils de type Stern-Gerlach. On montre que la théorie de dBB explique également cette expérience. De plus, la nouvelle interprétation causale que nous proposons corrige les défauts de l'interprétation bohmienne précédente et permet de décomposer la fonction d'onde des deux particules intriquées par deux fonctions d'ondes de particule unique. 


\section{Abstract}

\section{How can we understand the spin measure and the EPR-B experience? A causal interpretation}

Spin is an internal degree of freedom of any particle with no equivalent in classical mechanics. To understand its reality, in this paper, we go back to the Stein-Gerlach experiment which in 1922 revealed the 1/2 spin of the electron. This experiment is the archetype of measurement in quantum mechanics. It summarizes the so called problem of measurement (Schroedinger's cat). The spatial and temporal solution of Pauli's equation is necessary in order to understand the numerous subtleties of this experiment. Especially, it obliges to consider the spatial extension of the wave function. We show that the De Broglie-Bohm theory (dBB), which defines a reality underlying the usual quantum mechanics by adding hidden variables (particles positions), gives a causal and realistic explanation of this experiment. The David Bohm version of the EinsteinPodolsky-Rosen (EPR-B) experiment which highlights the non locality of a pair particles entangled by their spin uses Stein-Gerlach types of machines. We show that the dBB theory also explains this experiment. Moreover, our proposed new causal interpretation corrects the defects of the former Bohm interpretation. It allows to decompose the wave function of the two entangled particles in two unique particle wave functions.

\section{Expérience de Stern et Gerlach}

En étudiant en 1921 et 1922 un jet d'atomes d'argent traversant l'entrefer d'un aimant où règne un champ magnétique fortement inhomogène, Otto Stern et Walther Gerlach [10] mettent en évidence un résultat expérimental qui contredit la prévision théorique classique de l'époque : le faisceau, au lieu de s'élargir, se sépare en deux taches distinctes d'intensité identique. Nous supposerons comme schématisé par la figure I que le champ magnétique B est dirigé selon l'axe $O z$ et que le faisceau atomique arrive perpendiculairement à cet axe (selon $O y$ ). La déviation est due au moment magnétique induit par le moment cinétique interne (spin) de l'électron le moins lié de l'atome d'argent.

\section{Résolution par l'équation de Pauli}

\section{I Source ponctuelle ou extension spatiale : spineur}

L'état d'une particule de spin $1 / 2$ est décrit par une fonction d'onde $\Psi(\mathbf{x}, \mathrm{t})$, appelée spineur de Pauli, qui admet deux composantes complexes $\Psi^{+}(\mathbf{x}, \mathbf{t})$ et $\Psi^{-}(\mathbf{x}, \mathbf{t})$. Au moment d'entrer dans le champ magnétique $(t=0)$, on peut associer à chaque atome d'argent un spineur correspondant à un état pur, comme dans de nombreux livres de cours de mécanique quantique $[9,5,16,2]$ : 


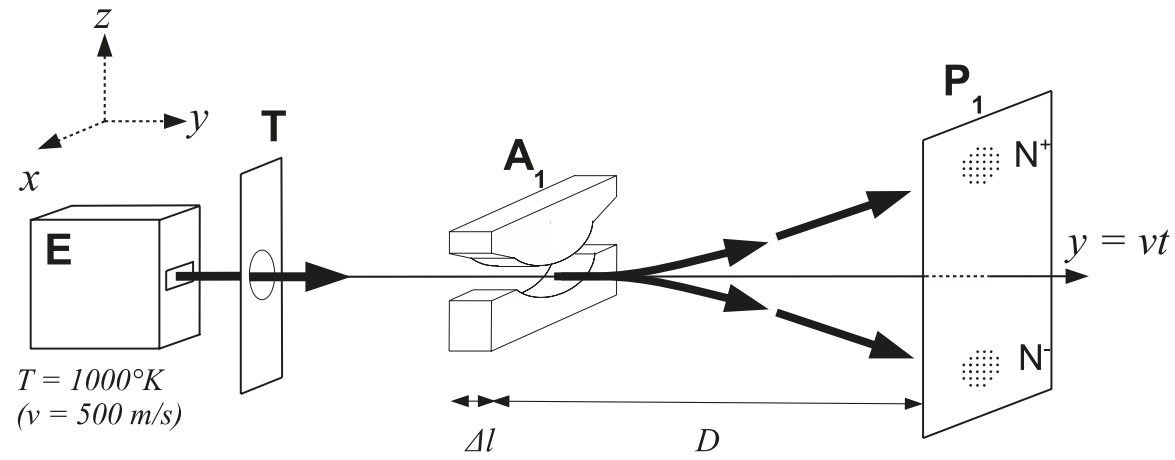

Figure 1. Schéma de l'expérience de Stern et Gerlach : un jet d'atomes d'argent, issu de la source (enceinte $\mathbf{E}$ chauffée, trou colmateur $\mathbf{T}$ ) traverse un champ magnétique inhomogène (aimant $\mathbf{A}_{1}$ ) puis se sépare en deux faisceaux distincts donnant sur la plaque $\mathbf{P}_{\mathbf{1}}$ deux taches distinctes d'intensité identique $N^{+}$et $N^{-}$.

$$
\Psi_{0}=\Psi(t=0)=\left(\begin{array}{c}
\cos \frac{\theta_{0}}{2} e^{i \frac{\varphi_{0}}{2}} \\
\sin \frac{\theta_{0}}{2} e^{-i \frac{\varphi_{0}}{2}}
\end{array}\right)
$$

où $\theta_{0}$ et $\varphi_{0}$ sont les angles polaires caractérisant l'état pur (cf. figure 2). Les angles $\theta_{0}$ et $\varphi_{0}$ sont évidemment inconnus et chaque atome du faisceau a des angles $\left(\theta_{0}, \varphi_{0}\right)$ différents; on sait seulement que $\theta_{0} \in[0 ; \pi]$ et $\varphi_{0} \in[0 ; 2 \pi[$. Le faisceau traversant le champ magnétique est un mélange statistique d'états purs, c'est à dire un mélange aléatoire d'atomes dans des états purs différents.

Pour faire un calcul analytique et complet de l'expérience et démontrer la contextualité de la mesure du spin, on verra plus loin qu'il est absolument nécessaire de représenter une particule individuelle avec spin par une fonction d'onde (spineur) avec une extension spatiale comme la suivante :

$$
\Psi_{0}(x, z)=\left(2 \pi \sigma_{0}^{2}\right)^{-\frac{1}{2}} e^{-\frac{x^{2}+z^{2}}{4 \sigma_{0}^{2}}}\left(\begin{array}{c}
\cos \frac{\theta_{0}}{2} e^{i \frac{\varphi_{0}}{2}} \\
\sin \frac{\theta_{0}}{2} e^{-i \frac{\varphi_{0}}{2}}
\end{array}\right)
$$

En effet le faisceau d'atomes d'argent n'est pas ponctuel et il faut considérer les variables d'espace $\mathbf{x}$ (seul les variables de spin sont prises en compte dans $(I)$ ); on considère dans la formule (2) un faisceau gaussien suivant les axes $O z$ et $O x$ d'écart type $\sigma_{0}=10^{-4} \mathrm{~m}$. L'axe $O y$ est l'axe de propagation des atomes ; il est traité classiquement sachant que la vitesse des atomes dans cette direction est environ de $v_{y}=500 \mathrm{~m} / \mathrm{s}$, ce qui correspond à une température du four $\mathbf{E}$ de $1000 \mathrm{~K}$. 


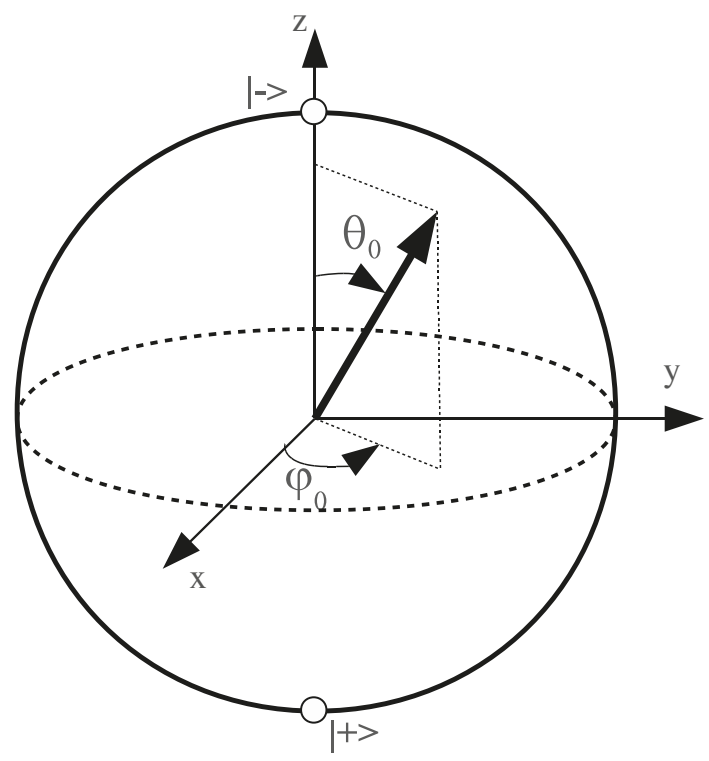

Figure 2. Orientation du spin d'un état pur. $\theta_{0}$ et $\varphi_{0}$ sont les angles polaires caractérisant le spineur. Ils correspondent aux angles de la sphère de Bloch.

Pour étudier l'évolution de la fonction d'onde pendant les mesures, nous n'utiliserons pas les postulats de la mécanique quantique liés à la mesure qui ne concernent que le résultat final, mais nous résoudrons l'équation de Schrödinger dépendante du temps ; l'équation de Schrödinger qui tient compte du spin I/2 est appelée l'équation de Pauli.

\section{2 Équation de Pauli}

L'évolution non relativiste du spineur $\Psi(\mathbf{x}, t)=\left(\begin{array}{l}\Psi^{+}(\mathbf{x}, t) \\ \Psi^{-}(\mathbf{x}, t)\end{array}\right)$ est donné par l'équation de Pauli. Pour une particule neutre (l'atome d'argent) et de spin $1 / 2$ l'équation de Pauli est :

$$
i \hbar \frac{\partial \Psi}{\partial t}(\mathbf{x}, t)=\left(-\frac{\hbar^{2}}{2 m} \nabla^{2}+\mu_{B} \mathbf{B} \boldsymbol{\sigma}\right) \Psi(\mathbf{x}, t)
$$

où $m=1.8 \times 10^{-25} \mathrm{~kg}$ est la masse de l'atome d'argent, $\mathbf{B}$ est le champ magnétique, $\mu_{B}=e \hbar / 2 m_{e}$ est le magnéton de Bohr et $\sigma=\left(\sigma_{x}, \sigma_{y}, \sigma_{z}\right)$ correspondent aux trois matrices de Pauli.

Sans l'extension spatiale initiale, la résolution spatiale de l'équation de Pauli est impossible et nous perdons la possibilité de tenir compte de l'évolution du spin durant le processus de mesure $[17,4,7,3]$. En effet, l'évolution différente des deux composantes du spineur joue un rôle clé dans la compréhension du processus de mesure. 


\subsection{Solution sans l'équation de Pauli et avec les postulats de la mesure}

II est très facile d'inférer le résultat de la mesure sans avoir recours à l'équation de Pauli en utilisant les postulats de la mesure. On réécrit le spineur initial sans extension spatiale $\Psi_{0}{ }^{1}$ de l'équation (I) sous la forme :

$$
\Psi_{0}=\cos \frac{\theta_{0}}{2} e^{i \frac{\varphi_{0}}{2}}|+\rangle+\sin \frac{\theta_{0}}{2} e^{-i \frac{\varphi_{0}}{2}}|-\rangle,
$$

où $|+\rangle=\left(\begin{array}{l}1 \\ 0\end{array}\right)$ et $|-\rangle=\left(\begin{array}{l}0 \\ 1\end{array}\right)$ sont les vecteurs propres de l'opérateur $\sigma_{z}$. D’après les postulats de la mesure, $\left|\cos \frac{\theta_{0}}{2} e^{i \frac{\varphi_{0}}{2}}\right|^{2}=\cos ^{2} \frac{\theta_{0}}{2}$ est la probabilité de mesurer le spin $+\hbar / 2$ (valeur propre de l'opérateur de projection $S_{z}=\hbar / 2 \sigma_{z}$ ) et $\sin ^{2} \theta_{0} / 2$ celle de mesurer le spin $-\hbar / 2$. Le $\theta_{0}$ étant inconnu au sortir de la source et sachant que chaque atome a un $\theta_{0}$ différent compris entre 0 et $\pi$, la probabilité de mesurer le spin $+1 / 2$, ne connaissant pas le spineur initial de l'atome incident, est égale à : $\int_{0}^{\pi} \cos ^{2} \frac{\theta_{0}}{2} d \theta_{0}=0.5$. Celle de mesurer le spin - I/2 est égale à : $\int_{0}^{\pi} \sin ^{2} \frac{\theta_{0}}{2} d \theta_{0}=0.5$. On retrouve donc les deux taches distinctes d'intensité identique $N^{+}$et $N^{-}$. On comprend aisément l'intérêt pratique des postulats de la mesure : ils permettent de retrouver simplement et rapidement les résultats expérimentaux ; cependant ils ne permettent pas une compréhension précise du phénomène de mesure.

\section{Théorie de de Broglie-Bohm}

Dans la théorie de de Broglie-Bohm, la fonction d'onde ne représente pas complètement l'état d'une particule quantique et il est nécessaire d'ajouter à sa description la position de la particule $X(t)$. L'évolution du spineur est toujours donné par l'équation de Pauli (3) et l'évolution de sa position est donnée par la formule de [4, 17] :

$$
\frac{d X(t)}{d t}=\left.\frac{\hbar}{2 m \rho} \operatorname{Im}\left(\Psi^{\dagger} \nabla \Psi\right)\right|_{\mathbf{x}=X(t)}
$$

où $\Psi^{\dagger}=\left(\Psi^{+*}, \Psi^{-*}\right)$ et $\rho=\Psi^{\dagger} \Psi$. L'atome est à la fois une onde et un corpuscule. Onde et corpuscule coexistent, la première guidant le second par l'équation (5), d'où le nom d’onde pilote donné par de Broglie en 1927.

Bohm et al. [4] définissent également s un champ de vecteurs de spin comme :

$$
\mathbf{s}(\mathbf{x}, t)=\frac{\hbar}{2 \rho} \Psi^{\dagger}(\mathbf{x}, t) \sigma \Psi(\mathbf{x}, t)=\frac{\hbar}{2}(\sin \theta \sin \varphi, \sin \theta \cos \varphi, \cos \theta)
$$

(I) Le raisonnement est similaire que l'on considère ou pas l'extension spatiale. C'est d'ailleurs la raison pour laquelle elle n'est souvent pas considérée. 
Le vecteur de spin d'une particule individuelle est évaluée le long de sa trajectoire comme étant : $\mathbf{s}=\mathbf{s}(X(t), t)$.

\section{I État pur}

On résout l'équation de Pauli pour un atome unique correspondant à un état pur avec $\left(\theta_{0}, \phi_{0}\right)$. Dans l'espace libre, au sortir du champ, au temps $t+\Delta t\left(t \geq 0\right.$ et $\Delta t=\Delta l / v_{y}$ étant la durée de traversée du champ), on obtient [II] :

$$
\Psi(x, z, t+\Delta t) \simeq\left(2 \pi \sigma_{0}^{2}\right)^{-\frac{1}{2}} e^{-\frac{x^{2}}{2 \sigma_{0}^{2}}}\left(\begin{array}{c}
\cos \frac{\theta_{0}}{2} e^{-\frac{(z-z \Delta-u t)^{2}}{2 \sigma_{z}^{2}}} e^{i \frac{m u z+\hbar \varphi_{+}}{\hbar}} \\
i \sin \frac{\theta_{0}}{2} e^{-\frac{(z+z \Delta+u t)^{2}}{2 \sigma_{z}^{2}}} e^{i \frac{-m u z+\hbar \varphi_{-}}{\hbar}}
\end{array}\right)
$$

où

$$
z_{\Delta}=\frac{\mu_{B} B_{0}^{\prime}(\Delta t)^{2}}{2 m}=10^{-5} m, \quad u=\frac{\mu_{B} B_{0}^{\prime}(\Delta t)}{m}=1 \mathrm{~m} / \mathrm{s}, \quad \mathbf{B}=\left(\begin{array}{c}
B_{0}^{\prime} x \\
0 \\
B_{0}-B_{0}^{\prime} z
\end{array}\right)
$$

et où $\varphi-$ et $\varphi+$ sont des constantes. La figure 3 représente $\rho(z, t)=\int \Psi^{\dagger}(x, z, t) \Psi(x, z, t) d x$, la densité de probabilité de présence de l'atome d'argent pour les valeurs $\theta_{0}=\pi / 3$ et $\varphi_{0}=0$. L'axe $O y$, de propagation du jet, est en abscisse $\left(y=v_{y} t\right)$ et l'axe $O z$ en ordonnée (la variable $x$ n'est pas représentée car l'onde reste gaussienne selon cet axe). L'aimant $A_{1}$ est représenté sur la figure par les deux rectangles blancs, il est long de $\Delta l=1 \mathrm{~cm}$ et il y a $D=20 \mathrm{~cm}$ de parcours libre avant que l'atome soit mesurée sur l'écran de détection $P_{1}$. Les données numériques proviennent du livre de Cohen-Tannoudji et al. [5]. Pour le champ électromagnétique $\mathbf{B}$, on a : $B_{0}=5$ Tesla, $B_{0}^{\prime}=\left|\frac{\partial B}{\partial z}\right|=-\left|\frac{\partial B}{\partial x}\right|=10^{3}$ Tesla $/ \mathrm{m}$. La théorie de $\mathrm{dBB}$ complète la mécanique quantique habituelle car comme on le voit sur la figure $3 b$, elle montre comment la position initiale de la particule explique de façon causale la position finale habituellement justifié par la réduction du paquet d'onde (figure 3a). Dans ce cadre, il n'y a plus de problème de mesure car la continuité de la mécanique quantique vers la mécanique classique se fait de façon naturelle (pas de création de notion de position).

La figure 4 représente la densité $\rho(z, t)$ pour l'état pur $\theta_{0}=\pi / 2$ ainsi que six trajectoires d'atomes dont la position initiale a été tiré au hasard.

L'équation de Pauli ne prévoit que la probabilité $\rho$ du lieu de l'impact de l'atome. Sa position effective dans l'interprétation de Copenhague est sans explication : c'est un aléa fondamental d'un type nouveau par rapport à la mécanique classique. Albert Einstein critiqua cette vision des choses par sa célèbre formule : "Dieu ne joue pas aux dés ». D'après lui, les prédictions aléatoires de la mécanique quantique ne provenaient pas d'un aléa « vrai » ou fondamental de la physique, mais d'une incomplétude du modèle : il devait exister un modèle sous-jacent qui explique ces aléas. La théorie de dBB définit justement un modèle sous-jacent possible à la mécanique quantique en ajoutant la notion de 


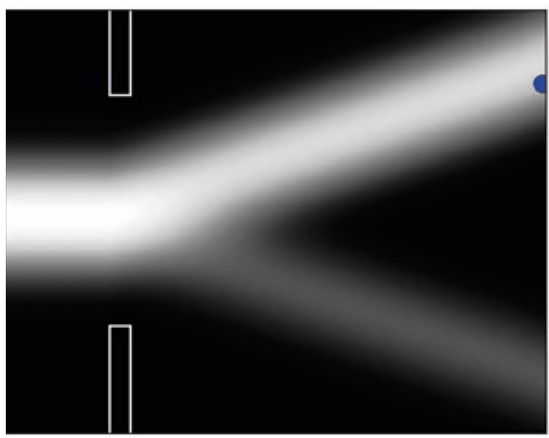

(a)

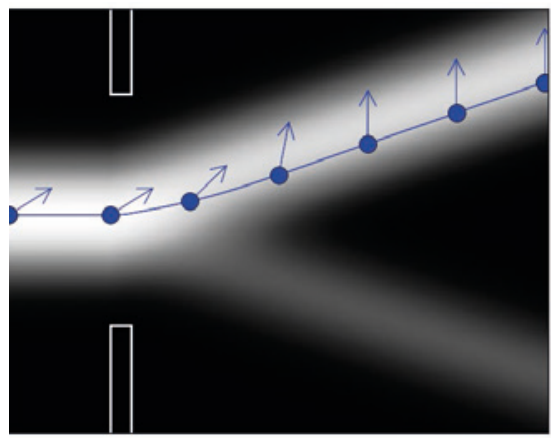

(b)

Figure 3. Les figures montrent l'évolution temporelle de la densité de probabilité de présence $\rho=\Psi^{\dagger} \Psi$ d'un atome d'argent dans un état pur $\theta_{0}=\pi / 3$ au cours de la traversée du champ magnétique (rectangles blancs). (a) Mécanique quantique orthodoxe : équation de Pauli + réduction du paquet d'onde (position finale). La notion de trajectoire n'existe pas; seule la position au moment de la mesure est définie à l'aide du postulat de réduction du paquet d'onde (point bleu). L'atome est soit une onde soit une particule (au moment de l'impact). (b) Théorie de dBB (mécanique quantique complétée) : équation de Pauli + position initiale. Les flèches indiquent l'orientation $\theta$ du vecteur spin s (initialement $\theta_{0}=\pi / 3$ ). La position de la particule existe avant la mesure; la particule suit alors une trajectoire déterministe et l'impact sur l'écran ne fait que révéler sa position. L'atome est à la fois une onde et une particule.

position des particules. L'atome est décrit à la fois par la fonction d'onde et par la position de son centre de gravité. II n'y a pas d'aléa fondamental, c'est la position initiale de l'atome (au sortir de la source) qui détermine son impact sur l'écran et, dans ce cas, « Dieu ne joue pas aux dés ». Cependant, du fait que la position initiale de l'atome n'est connue que statistiquement, la position finale ne peut, elle aussi, être connue en pratique que statistiquement. Les probabilités quantiques correspondent à notre ignorance précise du système.

Expérimentalement, on ne mesure pas directement le spin d'un atome mais la position $(\tilde{x}, \tilde{z})$ de l'impact de l'atome sur $P_{1}$. Si $\tilde{z} \in N^{+}$, le terme $\psi^{-}$de (7) est numériquement égal à zéro et le spineur $\Psi$ est proportionnel à $\left(\begin{array}{l}0 \\ 1\end{array}\right)$, l'un des vecteurs propres de $\sigma_{z}$. Si $\tilde{z} \in N^{-}$, le terme $\psi+$ de $(7)$ est numériquement égal a zéro et le spineur $\Psi$ est proportionnelle à $\left(\begin{array}{l}0 \\ 1\end{array}\right)$, l'autre vecteur propre de $\sigma_{z}$. Par conséquent, la mesure du spin correspond à une valeur propre de l'opérateur de spin $S_{z}=\hbar / 2 \sigma_{z}$. C'est une démonstration du postulat de quantification par la théorie de $\mathrm{dBB}$.

L'équation (7) donne les probabilités $\cos ^{2} \theta_{0} / 2$ (resp. $\left.\sin ^{2} \theta_{0} / 2\right)$ de mesurer la particule dans l'état de spin $+\hbar / 2($ resp. $-\hbar / 2)$. C'est une démonstration du postulat de la décomposition spatiale par la théorie de dBB. La valeur « mesurée » n'est pas une valeur préexistante. Elle est contextuelle et conforme au théorème de Kochen et Specker [13]. 


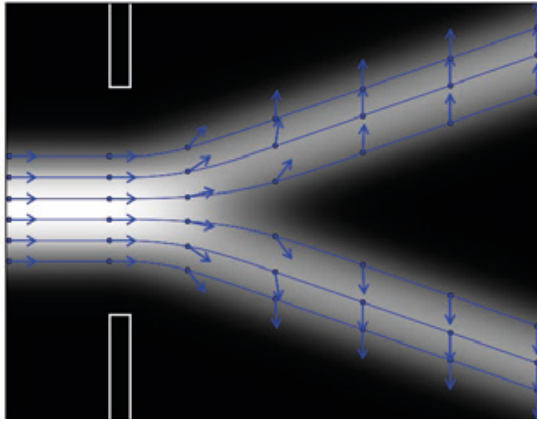

Figure 4. Six trajectoires d'atomes d'argent issues d'un même état pur $\theta_{0}=\pi / 2$. Les flèches indiquent l'évolution $d u$, initialement tous les $\theta=\pi / 2$.

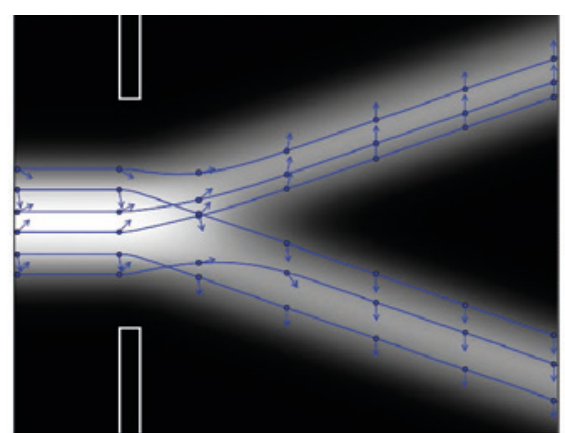

Figure 5. Six trajectoires d'atomes d'argent issues d'un mélange statistique d'états purs. Les flèches initiales indiquent le $\theta_{0}$ de l'état pur de chaque particule.

\subsection{Mélange statistique d'états purs}

Puisque le faisceau d'atomes d'argent est un mélange d'états purs, la densité atomique $\rho(z, t+\Delta t)$ est déterminée en intégrant $\rho(\mathrm{x}, \mathrm{z}, t+\Delta t)$ sur $x$ et $\operatorname{sur}\left(\theta_{0}, \varphi_{0}\right)$ soit :

$$
\rho(z, t+\Delta t)=\left(2 \pi \sigma_{0}^{2}\right)^{-\frac{1}{2}} \frac{1}{2}\left(e^{-\frac{\left(z-z_{\Delta}-u t\right)^{2}}{2 \sigma_{0}^{2}}}+e^{-\frac{\left(z+z_{\Delta}+u t\right)^{2}}{2 \sigma_{0}^{2}}}\right) .
$$

La figure 5 représente ce $\rho(z, t)$ ainsi que six trajectoires d'atomes dont la position initiale et l'orientation du spineur initial $\theta_{0}$ a été tiré au hasard. On notera la différence avec les résultats de la figure 4 pour l'état pur $\theta_{0}=\pi / 2$ qui donne cependant statistiquement les mêmes résultats.

\section{Expérience d'Einstein-Podolsky-Rosen-Bohm}

La figure 6 présente la version de David Bohm de l'expérience Einstein-Podolsky-Rosen (EPR-B). Une source $S$ crée en $O$ une paire d'atomes (d'argent) identiques $\mathrm{A}$ et $\mathrm{B}$, intriqués par leur spin avec des spins opposés. Les atomes $A$ et $B$ se séparent suivant l'axe $O y$ dans des directions opposées ( $\mathrm{A}$ avec la vitesse $v_{y}$ et $\mathrm{B}$ avec la vitesse $-v_{y}$ ), et rencontrent deux appareils identiques de type Stern-Gerlach $\mathbf{E}_{\mathrm{A}}$ et $\mathrm{E}_{\mathrm{B}}$.

L'aimant $\mathbf{E}_{\mathrm{A}}$ 《 mesure » (ou plutôt redresse) le spin de $\mathrm{A}$ dans la direction $\mathrm{O} z$ et l'aimant $\mathbf{E}_{\mathrm{B}}$ « mesure » (ou redresse) le spin de $\mathrm{B}$ dans la direction $O z^{\prime}$, direction obtenue après une rotation d'un angle $\delta$ autour de l'axe $O y$. Les deux « mesures » peuvent être simultanées ou effectuées l'une après l'autre, comme on va le considérer dans cet article. Cela n'a pas 


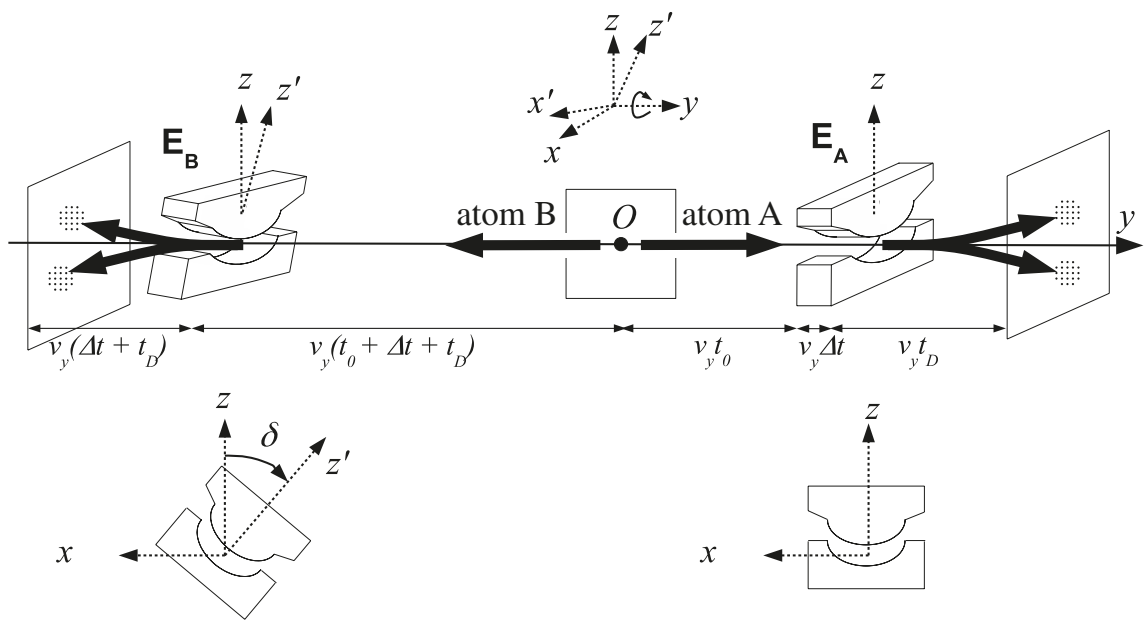

Figure 6. Schéma de l'expérience de EPR-B : une paire d'atome d'argent intriquée par leur spin traverse chacun à leur tour des aimants orientés dans des directions décalées de $\delta$.

d'importance théorique mais les deux appareils de mesure doivent être espacés d'une distance suffisamment grande pour qu'il n'y est pas de communications infra-lumineuses possibles entre les deux mesures. La corrélation des deux mesures de spin de $A$ et $B$ viole les inégalités de Bell et démontre l'existence d'une influence non locale, c'est-à-dire supra-lumineuse entre les deux spins des atomes d'argent.

\section{I État singulet : spineur initial}

Dans la plupart des papiers concernant l'expérience EPR-B, la fonction d'onde initiale du système quantique composé des deux particules intriquées est le spineur singlet :

$$
\Psi_{0}=\frac{1}{\sqrt{2}}\left(\left|+{ }_{A}\right\rangle\left|-{ }_{B}\right\rangle-\left|-{ }_{A}\right\rangle\left|+{ }_{B}\right\rangle\right)
$$

où $| \pm A\rangle$ (resp. $| \pm B\rangle$ ) sont les vecteurs propres de l'opérateur de spin $S_{z_{A}}$ (resp. $S_{z B}$ ) dans la direction $O z$ relatif à la particule A : $S_{z A}| \pm A\rangle= \pm(\hbar / 2)| \pm A\rangle$ (resp. B : $\left.S_{z B}| \pm B\rangle= \pm(\hbar / 2)| \pm B\rangle\right)$. En réalité, la fonction d'onde singulet initiale, comme pour le cas précédant, a une extension spatiale :

$$
\Psi_{0}\left(\mathbf{r}_{A}, \mathbf{r}_{B}\right)=\frac{1}{\sqrt{2}} f\left(\mathbf{r}_{A}\right) f\left(\mathbf{r}_{B}\right)\left(\left|+{ }_{A}\right\rangle\left|-{ }_{B}\right\rangle-\left|-{ }_{A}\right\rangle\left|+_{B}\right\rangle\right)
$$

où $\mathbf{r}=(x, z)$ et $f(\mathbf{r})=\left(2 \pi \sigma_{0}^{2}\right)^{-\frac{1}{2}} e^{-\frac{x^{2}+z^{2}}{4 \sigma_{0}^{2}}}$. 


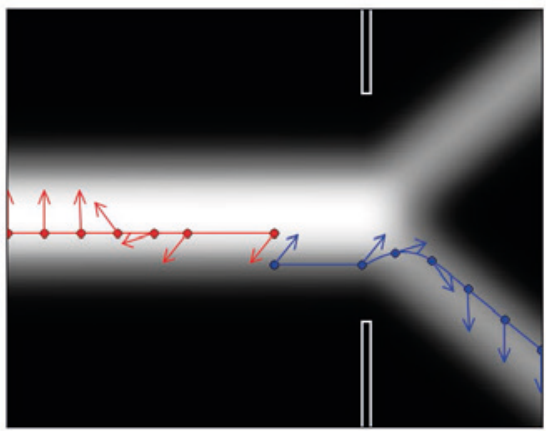

Figure 7. Première mesure : "mesure» du spin de A. Au centre les deux atomes intriqués $A$ (bleu) et $B$ (rouge) sont créés avec des spins opposés. La particule A part à droite et traverse l'aimant $\mathbf{E}_{\mathrm{A}}$, elle se comporte de la même façon que si elle n'était pas intriquée. La particule B part à gauche et ne traverse rien mais son spin se redresse pour être toujours en opposition avec celui de $A$.

Il est possible de retrouver cette fonction singulet (II) à partir du principe de Pauli. Pour cela, nous supposons qu'à l'instant de la création des deux particules intriquées $A$ et $\mathrm{B}$, chacune des particules a une fonction d'onde initiale $\Psi_{0}^{A}\left(\mathbf{r}_{A}, \theta_{0}^{A}, \varphi_{0}^{A}\right)$ et $\Psi_{0}^{B}\left(\mathbf{r}_{B}, \theta_{0}^{B}, \varphi_{0}^{B}\right)$ similaire à (2) mais avec des spins opposées : $\theta_{0}^{B}=\pi-\theta_{0}^{A}, \varphi_{0}^{B}=\varphi_{0}^{A}-\pi$. Si alors on applique le principe de Pauli qui stipule que la fonction d'onde d'un objet à deux corps doit être antisymétrique ; il faut écrire :

$$
\begin{aligned}
& \Psi_{0}\left(\mathbf{r}_{A}, \theta_{A}, \varphi_{A}, \mathbf{r}_{B}, \theta_{B}, \varphi_{B}\right) \\
& =\Psi_{A}^{0}\left(\mathbf{r}_{A}, \theta_{A}, \varphi_{A}\right) \Psi_{B}^{0}\left(\mathbf{r}_{B}, \theta_{B}, \varphi_{B}\right)-\Psi_{A}^{0}\left(\mathbf{r}_{B}, \theta_{B}, \varphi_{B}\right) \Psi_{B}^{0}\left(\mathbf{r}_{A}, \theta_{A}, \varphi_{A}\right) \\
& =-e^{i \varphi_{A}} f\left(\mathbf{r}_{A}\right) f\left(\mathbf{r}_{B}\right)\left(\left|+_{A}\right\rangle\left|-{ }_{B}\right\rangle-\left|-{ }_{A}\right\rangle\left|+_{B}\right\rangle\right)
\end{aligned}
$$

qui est l'état singulet avec extension spatiale (II) à un facteur près (voir [12] pour plus de détail). De nouveau cette extension spatiale est essentielle pour résoudre correctement l'équation de Pauli en espace. De plus, dans la théorie de dBB, l'extension spatiale est nécessaire pour tenir compte de la position de l'atome dans sa fonction d'onde.

\subsection{Résultats}

Sur la figure 7, on observe une paire d'atomes intriqués lors de la première « mesure » par un appareil de Stern-Gerlach. On montre mathématiquement [12] que la première particule « mesurée », la particule A, se comporte dans le premier appareil de Stern-Gerlach de la même façon que si elle n'était pas intriquée. Durant la « mesure » de $A$, la densité de la particule B évolue aussi comme si elle était non intriquée [12]. Ces deux propriétés pourront être testées expérimentalement dès que l'expérience EPR-B avec des atomes sera réalisable. La particule $B$ part vers la gauche et, durant la « mesure » de $A$, son spin se redresse pour être toujours en opposition avec celui de la particule $A$ [12]. Lorsque la particule $B$ 
entre dans le second aimant $\mathbf{E}_{\mathrm{B}}$ l'orientation de son spin $\theta$ par rapport à $O z$ sera soit 0 soit $\pi$; c'est-à-dire orienté selon l'axe $O z^{\prime}$ du champ de $\mathbf{E}_{\mathrm{B}}$, soit de $\delta$ soit de $\pi-\delta$. L'orientation de $\mathrm{B}$ est de $\delta$ si le spin de $\mathrm{A}$ a été «mesuré » down et elle est de $\pi-\delta$ si le spin de $\mathrm{A}$ a été " mesuré » up. La seconde mesure est une « mesure » de type Stern-Gerlach comme au début de l'article avec des orientations particulières. On retrouve alors parfaitement les résultats de la mécanique quantique et la violation des inégalités de Bell.

\section{Conclusion}

Nous avons montré que la théorie de $\mathrm{dBB}$, qui utilise uniquement la résolution de l'équation de Pauli, donne les mêmes résultats statistiques que la mécanique quantique habituelle pour les expériences de Stern-Gerlach et EPR-B. Elle propose une claire interprétation de la mesure du spin en démontrant les postulats de la mesure et le postulat de réduction du paquet d'onde.

L'expérience de Stern et Gerlach n'est pas la mesure de la projection du spin selon l'axe $\mathrm{O} z$, mais le redressement de l'orientation du spin soit dans la direction du gradient du champ magnétique, soit dans la direction opposée. Le résultat dépend de la position de la particule dans la fonction d'onde. C'est une explication simple de la non contextualité de la mesure du spin le long de différents axes. La durée de la mesure est le temps nécessaire à la particule pour redresser son spin dans la direction finale. La valeur « mesurée » (le spin) n'est pas une valeur préexistante comme la masse et la charge de la particule mais une valeur contextuelle conforme au théorème de Kochen et Specker.

La particule quantique possède une position locale comme une particule classique, mais possède également un comportement non-local du à la fonction d'onde.

La réalité de la non-localité, c'est-à-dire l'existence d'une action à distance supralumineuse, a été validée par les expériences d'Alain Aspect [I] et de ses successeurs Nicolas Gisin [18] et Anton Zeilinger [14]. Cette action est en contradiction avec l'interprétation d'Einstein (de 1905) de la relativité restreinte. Faut-il abandonner cette interprétation au profit de celle de Lorentz-Poincaré comme le suggère Karl Popper dans sa préface [15] ?

\footnotetext{
"L'expérience d'Aspect serait la première expérience cruciale visant à trancher entre les interprétations lorentzienne et einsteinienne des transformations de Lorentz »
}

La théorie de $\mathrm{dBB}$ permet de considérer la mécanique quantique comme déterministe et ainsi permet de lever la première difficulté pour rendre compatible mécanique quantique et relativité générale. Réhabiliter l'existence d'un référentiel privilégié, comme celui de Lorentz-Poincaré et comme l'éther d'Einstein [8] de 1920, est peut-être le chemin pour réconcilier ces deux théories. 
Le réel nous serait alors doublement voilé. Voilé en mécanique quantique, comme le pensait Bernard d'Espagnat [6] car la fonction d'onde est une variable cachée non locale, mais voilé aussi en relativité car le temps et l'espace que nous mesurons ne seraient qu'un temps et un espace apparents, l'espace vrai et le temps vrai du référentiel et du temps privilégiés nous étant encore inconnus.

\section{Références}

[I] Aspect, Alain, Grangier, Philippe, et Roger, Gérard. Experimental realization of Einstein-Podolsky-Rosen-Bohm Gedankenexperiment: a new violation of Bell's inequalities. Physical review letters, 1982, vol. 49, n², p. 91.

[2] Bellac, M. L. Quantum physics. Cambridge University Press, 2006.

[3] Bohm, D. et Hiley, B. The Undivided Universe. Routledge, 1993.

[4] Bohm, David, Schiller, Ralph, et Tiomno, Jayme. A causal interpretation of the Pauli equation (A). II Nuovo Cimento (1955-1965), 1955, vol. I, nI, p. 48.

[5] Cohen-Tannoudji, Claude, Diu, Bernard et Laloë, Frank. Quantum mechanics. Wiley, 1977.

[6] D'Espagnat, Bernard. Veiled Reality: An analysis of present-day quantum mechanical concepts. Westview Press, 2003.

[7] Dewdney, C., Holland, P.R. et Kyprianidis, A. A causal account of non-local Einstein-Podolsky-Rosen spin correlations. Journal of Physics A: Mathematical and General, 1987, vol. 20, n¹4, p. 47I7. https://doi.org//0.1088/0305-4470/20//4/016

[8] Einstein, Albert. Ether and the Theory of relativity, 1920.

[9] Feynman, Richard P., Leighton, Robert B. et Sands, Matthew. The Feynman lectures on Physics, volume II. Addison-Wesley, Reading, MA, 1964.

[10] Gerlach, Walther et Stern, Otto. Der experimentelle nachweis der richtungsquantelung im magnetfeld. Zeitschrift für Physik, 1922, vol. 9, nI, p. 349.

[II] Gondran, Michel et Gondran, Alexandre. A complete analysis of the Stern-Gerlach experiment using Pauli spinors. 2005. https://arxiv.org/abs/quant-ph/05/1276.

[12] Gondran, Michel et Gondran, Alexandre. Replacing the singlet spinor of the EPR-B experiment in the configuration space with two single-particle spinors in physical space. Foundations of Physics, 2016, vol. 46, n9, p. 1109.

[13] Kochen, Simon et Specker, Ernst P. The problem of hidden variables in quantum mechanics. Journal of Mathematics and Mechanics, 1967, vol. 17, nI, p. 59. 
[14] Jian-Wei, Pan, Bouwmeester, Dik, Daniell, Matthew, Weinfurter, Harald et Zellinger, Anton. Experimental test of quantum nonlocality in three-photon Greenberger-Horne-Zeilinger entanglement. Nature, 2000, vol. 403, n6769, p. 515.

[15] Popper, Karl R. Quantum theory and the schism in Physics: From the postscript to the logic of scientific discovery. Edited by William Warren Bartley, III. London, 1982.

[16] Sakurai, Jun John et Napolitano, Jim. Modern quantum mechanics. Addison-Wesley, 201I.

[17] Takabayasi, Takehiko. On the Hydrodynamical Representation of non-relativistic spinor equation. Progress of Theoretical Physics, 1954, vol. 12, n6, p. 810.

[18] Tittel, Wolfgang, Brendel, Jürgen, Zbinden, Hugo et Gisin, Nicolas. Violation of Bell inequalities by photons more than $10 \mathrm{~km}$ apart. Physical Review Letters, 1998, vol. 81, n¹7, p. 3563. https://arxiv.org/abs/quant-ph/9806043 
